\title{
Estudo da neuropatia periférica em pacientes com doença de Parkinson: Revisão de
}

\section{literatura}

\author{
Study of peripheral neuropathy in patients with Parkinson disease: Literature review \\ Estudio de la neuropatía periférica en pacientes con enfermedad de Parkinson: Revisión de la lite-
}

ratura

Recebido: 04/06/2021 | Revisado: 10/06/2021 | Aceito: 11/06/2021 | Publicado: 24/06/2021

\author{
Antônia Viana \\ ORCID: https://orcid.org/0000-0002-6061-5029 \\ Universidade do Estado do Rio de Janeiro. Brasil \\ E-mail: ivoneviana@yahoo.com.br \\ João Santos Pereira \\ ORCID: https://orcid.org/0000-0002-3242-8461 \\ Universidade do Estado do Rio de Janeiro. Brasil \\ E-mail: jspereira.md@uol.com.br \\ Robson Vital \\ ORCID: https://orcid.org/0000-0003-4713-1203 \\ Universidade do Estado do Rio de Janeiro. Brasil \\ E-mail: rt.vital@uol.com.br
}

\begin{abstract}
Resumo
Nas últimas décadas, tem-se observado a presença de neuropatia periférica (NP) em pacientes com doença de Parkinson (DP), principalmente naqueles em tratamento crônico com levodopa (LD). A associação entre a NP e dose cumulativa de LD, os baixos níveis séricos de vitamina B12 e altos níveis plasmáticos de homocisteína e ácido metilmalônico tornaram-se evidentes. Objetivo: Identificar os fatores de risco relacionados a presença de Neuropatia periférica na Doença de Parkinson. Método: Utilizou-se as bases de dados Scielo, Pub Med e LILACS com as palavras-chave "Doença de Parkinson", "neuropatia periférica", "levodopa", "nervos periféricos" nos idiomas inglês, espanhol e português dos últimos 10 anos. Resultados: A maioria dos estudos de indivíduos com DP em uso crônico de LD, com altos níveis de ácido metilmalônico e homocisteína e deficiência de vitamina B12, apresentaram elevada prevalência de NP, predominando a forma sensório-motora com denervação axonal. Por outro lado, a presença de alfa sinucleina nos nervos periféricos pode determinar um processo degenerativo no sistema nervoso periférico. Conclusões: A neuropatia periférica em indivíduos com DP, além de relacionada a progressão e gravidade da doença, pode envolver outros fatores de risco como o uso prolongado de LD, deficiência de vitamina B12 e altos níveis séricos de homocisteína e ácido metilmalônico. A presença de alfa-sinucleina cutânea e nos nervos periféricos preconiza envolvimento no processo degenerativo periférico. Sugerem-se estudos prospectivos correlacionando o comprometimento do sistema nervoso periférico em pacientes com DP e o envolvimento de distintos fatores de risco com a progressão e agravamento da sintomatologia motora nesta doença.
\end{abstract}

Palavras-chave: Doença de Parkinson; Neuropatia periférica; Fatores de risco.

\begin{abstract}
In the last decades, the presence of peripheral neuropathy (PN) has been observed in patients with Parkinson's disease (PD), especially in those under chronic treatment with levodopa (LD). The association between PN and the cumulative dose of LD, low serum levels of vitamin B12 and high plasma levels of homocysteine and methylmalonic acid become evident. Objective: To identify risk factors related to the presence of peripheral neuropathy in Parkinson's disease. Method: Scielo, Pub Med and LILACS databases were used with the keywords "Parkinson's disease", "peripheral neuropathy", "levodopa", "peripheral nerves" in the English, Spanish and Portuguese languages of the last 10 years. Results: Most studies of individuals with PD in chronic use of LD, with high levels of methylmalonic acid and homocysteine and vitamin B12 deficiency, showed a high prevalence of PN, with a predominance of the sensorimotor form with axonal denervation. On the other hand, the presence of alpha synuclein in the peripheral nerves can determine a degenerative process in the peripheral nervous system. Conclusions: Peripheral neuropathy in individuals with PD, in addition to being related to disease progression and severity, may involve other risk factors such as prolonged use of LD, vitamin B12 deficiency and high serum levels of homocysteine and methylmalonic acid. The presence of alphasynuclein in the skin and in peripheral nerves suggests involvement in the peripheral degenerative process. Prospective studies correlating the involvement of the peripheral nervous system in patients with PD and the involvement of different risk factors with the progression and worsening of motor symptoms in this disease are suggested.
\end{abstract}

Keywords: Parkinson disease; Peripheral neuropathy; Risk factors. 


\section{Resumen}

En las últimas décadas se ha observado la presencia de neuropatía periférica (NP) en pacientes con enfermedad de Parkinson (EP), especialmente en aquellos en tratamiento crónico con levodopa (LD). Se hace evidente la asociación entre la NP y la dosis acumulada de LD, los niveles séricos bajos de vitamina B12 y los niveles plasmáticos altos de homocisteína y ácido metilmalónico. Objetivo: Identificar factores de riesgo relacionados con la presencia de neuropatía periférica en la enfermedad de Parkinson. Método: Se utilizaron las bases de datos Scielo, Pub Med y LILACS con las palabras clave "enfermedad de Parkinson", "neuropatía periférica", "levodopa", "nervios periféricos" en los idiomas inglés, español y portugués de los últimos 10 años. Resultados: La mayoría de estudios de individuos con EP en uso crónico de $\mathrm{LD}$, con altos niveles de ácido metilmalónico y deficiencia de homocisteína y vitamina B12, mostraron una alta prevalencia de NP, con predominio de la forma sensitivomotora con denervación axonal. Por otro lado, la presencia de alfa sinucleína en los nervios periféricos puede determinar un proceso degenerativo en el sistema nervioso periférico. Conclusiones: La neuropatía periférica en individuos con EP, además de estar relacionada con la progresión y severidad de la enfermedad, puede involucrar otros factores de riesgo como el uso prolongado de LD, deficiencia de vitamina B12 y niveles séricos elevados de homocisteína y ácido metilmalónico. La presencia de alfasinucleína en la piel y en los nervios periféricos sugiere una participación en el proceso degenerativo periférico. Se sugieren estudios prospectivos que correlacionen la afectación del sistema nervioso periférico en pacientes con EP y la implicación de diferentes factores de riesgo con la progresión y empeoramiento de los síntomas motores en esta enfermedad.

Palabras clave: Enfermedad de Parkinson; Neuropatía periférica; Factores de riesgo.

\section{Introdução}

A doença de Parkinson idiopática (DP) é uma doença neurodegenerativa crônica e progressiva que acomete o sistema nervoso central, sendo a principal causa de parkinsonismo. Considerada como a segunda doença neurodegenerativa mais frequente, após Alzheimer, afetando todas as populações, independente da etnia, classe social e profissão, havendo certa predominância no sexo masculino. Estudos evidenciam que acomete cerca de $0,3 \%$ da população mundial, sendo que sua prevalência aumenta exponencialmente com a idade, ocorrendo maior pico, em torno de 2-3\%, acima de 65 anos (Poewe et al, 2017; Tysnes \& Storstein, 2017). Entretanto, cerca de 5 a 10\% pode inciciar os sintomas parkinsonianos antes dos 40 anos (Radhakrishnan \& Goyal, 2018).

Embora não existam estatísticas nacionais, segundo a Associação Brasil Parkinson presume-se que existam cerca de 200.000 indivíduos com doença de Parkinson no Brasil. Estudo regional, realizado na cidade de Bambuí (MG), tendo como objetivo verificar a prevalência de parkinsonismo e suas causas em pessoas acima de 63 anos de idade, encontrou esta sintomatologia em 7,2\% dos participantes. A causa mais frequente foi a doença de Parkinson idiopática com 3,3\%, seguindo-se de parkinsonismo induzido por drogas com 2,7\% (Barbosa et al. 2006). O diagnóstico desta doença é essencialmente clínico e baseia-se na presença de alterações motoras como bradicinesia, rigidez e tremor de repouso. A presença dos denominados sintomas não motores, envolvendo distintas disfunções, como: hiposmia, distúrbios do sono REM, sintomas depressivos, ansiedade, disfunção autonômica (principalmente hipotensão ortostática, disfunção urogenital, constipação intestinal e hiperidrose), fadiga, sintomas sensitivos e dor, podem preceder o aparecimento dos sintomas motores clássicos em vários anos (Obeso et al., 2017; Poewe et al, 2017).

O principal substrato patológico para comprometimento da DP está na degeneração dos neurônios dopaminérgicos da pars compacta na substância negra (SN) com denervação no estriado. Sob o aspecto anatomopatológico, os neurônios dopaminérgicos reminiscentes na SN mostram a presença de inclusões de agregados proteicos de alfa-sinucleína, denominadas corpos de Lewy (Dickson, 2018).

Embora a maior preocupação para que se possa estabelecer o diagnóstico da DP, seja identificar o grau de comprometimento motor durante sua progressão, outros fatores poderão contribuir para que ocorra piora desses sintomas durante a progressão da doença. Apesar dos efeitos adversos da medicação, as flutuações motoras e a presença de algumas comorbidades associadas, como diabetes mellitus, hipertensão arterial, hipotiroidismo e até mesmo o envelhecimento, possam 
contribuir para piora da sintomatologia durante a progressão da doença, evidências têm mostrado o envolvimento do sistema nervoso periférico na gênese motora da doença durante sua progressão. Apesar desses achados não estejam sendo valorizados, nas últimas décadas estudos têm demonstrado alta prevalência de neuropatia periférica (NP) em pacientes com DP, principalmente naqueles em tratamento com levodopa (LD) à longo prazo (Cossu et al., 2016; Park et al., 2017). Estudo randomizado relacionando 500 indivíduos que apresentavam DP com a presença de neuropatia periférica idiopática, encontrou 34 casos (6,8\%) com esta associação. Destes, em 32 mostravam níveis anormais de homocisteína (hcy), ácido metilmalônico (AMM) e vitamina B12 (Toth et al., 2008). Em outro estudo, tipo caso-controle, foi encontrada NP, relacionada a deficiência de vitamina B12, em 38\% dos participantes do grupo DP (vs 8\% dos controles), por exposição cumulativa a levodopa e a duração da DP (Rajabally \& Martey, 2011).

Diante desses pressupostos, realizou-se esta revisão bibliográfica tendo como proposta identificar os principais fatores de risco desencadeantes de neuropatia periférica em pacientes com doença de Parkinson.

\section{Métodos}

Através desta revisão integrativa da literatura sobre doença de Parkinson e fatores de risco na neuropatia periférica procurou-se resumir os resultados do tema, abordando estudos experimentais e não experimentais, seguindo etapas de elaboração, definição das bases de dados, critérios de inclusão e exclusão dos estudos, seleção de informações, avaliação dos estudos, interpretação dos resultados e síntese de conhecimento (Mendes, Silveira, \& Galvão, 2008).

Foi utilizado o banco de dados Digital através do PubMed, Scielo e Lilacs, tendo como descritores "Doença de Parkinson" associada a um dos seguintes termos: "neuropatia periférica", "levodopa" e "nervo periférico" nos idiomas inglês, espanhol e português, sendo excluídos aqueles com informações ou metodologia pouco claras, resultados inconclusivos ou considerados como não relevantes para o tópico proposto. Foram selecionados e analisados estudos dos últimos 10 anos, com inclusão de dois artigos anteriores ao período, esclarecedores, considerados como importantes para o desenvolvimento desta pesquisa. O processo de seleção foi realizado em algumas etapas, obedecendo os objetivos propostos e os critérios de inclusão. Inicialmente foram considerados os títulos dos artigos, seguindo-se a leitura dos resumos. Utilizaram-se apenas artigos completos, disponíveis on-line. Excluiram-se editoriais, estudos publicados em anais de congresso, textos incompletos ou publicados em outros idiomas. Através de uma planilha realizou-se o registro das informações de cada artigo em relação ao título, autor, ano, metodologia, resultados e conclusões.

\section{Resultados}

Após busca inicial, utilizando as palavras-chave e considerando as bases de dados analisadas, de 56 artigos consultados, foram selecionados 36 para este estudo. Estes, após analisados, mostraram dois fatores causais importantes envolvendo o comprometimento da DP pela presença de neuropatia: o uso crônico de levodopa e a progressão da doença. Destes, 29 estudos, sendo 25 transversais, um de coorte prospectivo, um multicentrico e dois caso controle, encontram-se resumidos na Tabela 1. Nesta consulta estão incluidas 11 revisões de literatura consideradas relevantes. O número de participantes envolvidos em cada estudo variou de 14 a 330 participantes, com media de idade 63,2 +/- 14,8 anos. 
Tabela 1: Características gerais dos estudos selecionados.

\begin{tabular}{|c|c|c|c|c|}
\hline Autor/ano & $\begin{array}{l}\text { Delineamento do } \\
\text { estudo }\end{array}$ & Amostra & Objetivos do estudo & Resultados/conclusão \\
\hline Barbosa et al., 2006 & Estudo transversal & $\begin{array}{l}\text { Na fase I, } 1.186 \text { pessoas com mais de } 64 \text { anos } \\
\text { responderam a uma triagem de } 9 \text { perguntas questionário } \\
\text { para parkinsonismo. Na fase II, todos os indivíduos que } \\
\text { marcou } 2 \text { pontos no teste foram examinados } \\
\text { independentemente por pelo menos } 2 \text { médicos treinados } \\
\text { em distúrbios do movimento. }\end{array}$ & $\begin{array}{l}\text { Avaliar a prevalência de } \\
\text { parkinsonismo e suas causas } \\
\text { em uma coorte de indivíduos } \\
\text { com } 64 \text { anos anos ou mais em } \\
\text { Bambuí, uma cidade brasileira. }\end{array}$ & $\begin{array}{l}\text { A taxa de prevalência por } 100 \text { habitantes acima de } 64 \text { anos neste grupo foi } 7,2 \% \\
\text { para parkinsonismo de todos os tipos (n 86). As mais frequentes causas foram } \\
\text { DP idiopática e Parkinsonismo induzido por drogas, com taxas de prevalência } \\
\text { de 3,3\% (n 39) e 2,7\% (n 32), respectivamente. A prevalência de parkinsonismo } \\
\text { vascular foi de } 1,1 \% \text { (n 13). Foi encontrado } 1 \text { caso de Parkinsonismo pós- } \\
\text { traumático e outro de atrofia de múltiplos sistemas. }\end{array}$ \\
\hline Cossu et al., 2016 & Estudo transversal & $\begin{array}{l}\text { Foram avaliados } 197 \text { pacientes com DP. } 144 \text { foram } \\
\text { expostos à LD somente por mais de três anos (grupo } \\
\text { LELD); } 53 \text { utilizavam simultaneamente o Entacapone } \\
\text { por pelo menos dezoito meses (grupo LELD_ICOMT). } \\
\text { Foram submetidos à avaliação clínico-neurológica e } \\
\text { estudos de condução nervosa. }\end{array}$ & $\begin{array}{l}\begin{array}{l}\text { Determinar } \\
\text { inibidores }\end{array} \text { o } \begin{array}{c}\text { uso de } \\
\text { catecol-O- }\end{array} \\
\text { metiltransferase } \\
\text { podem reduzir o } \text { (ICOMT) } \\
\text { neuropatia induzida por LD na } \\
\text { DP. }\end{array}$ & $\begin{array}{l}\text { A prevalência de NP em pacientes do grupo LELD foi de } 19,4 \% \text {, versus } 5,7 \% \text { no } \\
\text { grupo LELD_ICOMT. Neste, a dose diária de LD e os níveis sérios de VB12 } \\
\text { foram significativamente mais altos e os níveis de Hcy foram significativamente } \\
\text { mais baixos comparado ao grupo LELD.Conclusão: Os ICOMT poderia ter um } \\
\text { efeito protetor no desenvolvimento da NP induzida pelo LD. Sua ação } \\
\text { provavelmente ocorre através do equilíbrio metabólico do ciclo da via do } \\
\text { carbono e é independente da duração e gravidade da DP e tempo da ingesta de } \\
\text { LD }\end{array}$ \\
\hline Toth et al., 2008 & Estudo transversal & $\begin{array}{l}\text { Foram avaliados } 500 \text { pacientes com DP e NP } \\
\text { sintomática sob o ponto de vista clínico, } \\
\text { eletrofisiológico e laboratorial. Essa população de } \\
\text { pacientes com DP com NP idiopática (DP-IPN) foi } \\
\text { comparada a um grupo de pacientes com DP sem PN } \\
\text { (apenas DP) e um grande grupo de pacientes sem DP } \\
\text { com NP idiopática (NIP) para anormalidades nos níveis } \\
\text { sérios de VB12, } \\
\text { homocisteína (Hcy) e AMM em jejum. }\end{array}$ & $\begin{array}{l}\text { Avaliar a presença de NP em } \\
\text { pacientes com DP. }\end{array}$ & $\begin{array}{l}\text { Dos } 49 \text { pacientes com DP com sintomas NP, } 34(69 \%) \text { apresentaram DP-NP e } \\
32 / 34(94 \%) \text { níveis anormais de Hcy ou MMA versus } 26 / 258(10 \%) \text { de } \\
\text { pacientes com NP. A dosagem cumulativa de LD ao longo da vida e os níveis de } \\
\text { MMA em jejum foram associados à gravidade da NP. A terapia com VB12 levou } \\
\text { a melhorias nos níveis de Hcy e MMA em todos grupos e NP em pacientes com } \\
\text { DP-NP. } \\
\text { Conclusão: NP em pacientes com DP pode estar associada à deficiência de } \\
\text { VB12. Alternativamente, a NP pode ser uma manifestação do SNP da DP. }\end{array}$ \\
\hline Rajabally et al., 2011 & Estudo transversal & $\begin{array}{l}\text { Incluidos } 37 \text { pacientes com DP em uso de LD de idade } \\
\text { média de } 67,9 \text { anos e duração média de doença de } 5,9 \\
\text { anos, e } 37 \text { pacientes controles sem DP, submetidos a } \\
\text { ENMG, exame neurológico e avaliados pela escala } \\
\text { Utah Early Neuropathy Scale (UENS) exames } \\
\text { laboratoriais. }\end{array}$ & $\begin{array}{l}\text { Avaliar a prevalência de NP, } \\
\text { bem como sua correlação com } \\
\text { a exposição cumulativa à LD e } \\
\text { os níveis de vitamina B12. }\end{array}$ & $\begin{array}{l}\text { Foram diagnosticados } 14 / 37 \text { pacientes com DP }(37,8 \%) \text { e } 3 / 37(8,1 \%) \text { controles } \\
\text { com NP.O escore UENS médio em pacientes com DP foi de } 4,43 \text { (DP } 3,71) \text { vs } \\
2,7 \text { (DP 2,54) em indivíduos controle.Oito dos } 14 \text { pacientes com DP }(57,1 \%) \\
\text { com NP apresentaram níveis de vitamina B12 de } 300 \mathrm{ng} / \mathrm{L} \text {, compatíveis com } \\
\text { deficiência. Conclusão: Há uma maior prevalência de NP em pacientes com DP. } \\
\text { Ocorreu correlação positiva entre exposição cumulativa à LD e baixos níveis de } \\
\text { vitamina B12, sugerindo que a NP pode estar relacionada à terapia com LD. }\end{array}$ \\
\hline Toth et al., 2010 & $\begin{array}{l}\text { Estudo de coorte } \\
\text { prospectivo }\end{array}$ & $\begin{array}{l}\text { Cinquenta e oito pacientes com DP foram selecionados } \\
\text { aleatoriamente e comparados com 58 controles } \\
\text { pareados por idade e sexo. Foram submetidos a } \\
\text { avaliação clinico-neurológica e avaliados por escalas- } \\
\text { Toronto Clinical Scoring System (TCSS) } 6 \text { e } \\
\text { Neuropathy Impairment Score (NIS) , exames } \\
\text { laboratoriais e ENMG. }\end{array}$ & $\begin{array}{l}\text { Avaliar a prevalência de NP em } \\
\text { pacientes com DP e sua } \\
\text { relação com o uso de LD e } \\
\text { níveis de ácido metilmalônico } \\
\text { (MMA) em jejum. }\end{array}$ & $\begin{array}{l}\text { Os sintomas sugestivos de NP foram presente em } 43 \% \text { dos pacientes com DPI e } \\
5 \% \text { dos controles. } 86 \% \text { dos pacientes estavam em uso de LD. Apenas } 5 / 8(9 \%) \\
\text { controles foram diagnosticados com NP. Os níveis de cobalamina foram } \\
\text { semelhante entre pacientes com DP-PN e DP-somente. Os níveis de Hcy e } \\
\text { MMA em jejum foram maiores em pacientes com DP-PN versus pacientes com } \\
\text { DP. Conclusão: Pacientes com DP têm maior prevalência de NP do que } \\
\text { controles. O uso de LD está associado à elevação do MMA e NP sensório- } \\
\text { motora em pacientes com DP. }\end{array}$ \\
\hline
\end{tabular}


Andreasson et al., Estudo transversal 2017

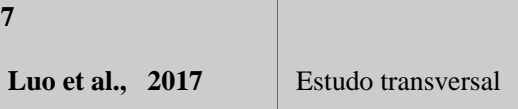

8

Park et al., 2017

Estudo transversal

\begin{tabular}{|c|c|c|}
\hline & & $\begin{array}{l}\text { de Dor neuropática coreano KNPQ). Foram ainda } \\
\text { submetidos à avaliaçãa laboratorial de vitamina B12, } \\
\text { Ácido metilmalônico (MMA) e homocisteína. Foram, a } \\
\text { seguir, estabelecidas correlações entre idade, duração da } \\
\text { DP, dose equivalente de LD , UPDRS III, VB12, } \\
\text { MMA e homocisteína. }\end{array}$ \\
\hline Vanta et al., 2019 & Estudo transversal & $\begin{array}{l}\text { Foram avaliados } 73 \text { pacientes, sendo } 50,7 \% \text { homens,de } \\
\text { idade média } 65,44 \pm 9,87 \text { com DP sem uma causa } \\
\text { previamente conhecida de NP usando escores clínicos } \\
\text { (UPDRS II e III e Toronto Clinical Scoring System), } \\
\text { avaliação dos níveis sérios de vitamina B12 e ácido } \\
\text { fólico, e estudos de condução nervosa para avaliar a } \\
\text { prevalência e características de NP. }\end{array}$ \\
\hline 10 & & \\
\hline Szadejk et al ., 2016 & Estudo transversal & $\begin{array}{l}\text { Foram avaliados } 102 \text { pacientes ( } 51 \text { com DP e } 51 \\
\text { controles saudáveis pareados por idade e sexo ) do } \\
\text { ponto de vista clínico-neurológico , submetidos a } \\
\text { escalas de NP e escalas para avaliar a severidade da } \\
\text { doença( UPDRS escala Hoehn-Yahr) e ENMG. }\end{array}$ \\
\hline
\end{tabular}

Descrever possíveis relações entre o metabolismo do ciclo da metionina e o desenvolvimento de NP em pacientes tratados com $\mathrm{LD}$ e de risco genticos através da e risco genéticos enotipagem das enzimas envolvidas.

Foram estudados 26 braços e 26 pernas de 28 casos de NP isolada induzida por homocisteína (IHIN),com idade média de $66,3 \pm 9,4$ anos \pm DP e masculino feminino $=12 / 16$, sendo submetidos a exam laboratoriais , estudo de condução nervosa e EMG

Avaliação clínico-neurológica 43 pacientes com DP. Quinze foram diagnosticados com NP de acordo com o sistema de pontuação clínica de Toronto (TCSS). A severidade da dor neuropática foi avaliada utilizando-se

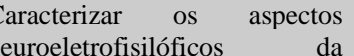
neuroeletrofisilóficos $\frac{d a}{\text { condução nervosa através da }}$ ENMG de extremidades superiores e inferiores de pacientes com alterações na IHIN.

Avaliar a relação entre os níveis séricos de vitamina B12 (VB12) e seu precursor MMA em pacientes com DP com dor neuropática. Avaliar a NP de fibras grossas em pacientes com DP e sua relação com a deficiência e vitamina B12 e exposição à LD.

Determinar a prevalência de $\mathrm{NP}$ independentemente de outras etiologias, e determinar a relação com fatores clínicos e demográficos em pacientes com DP tratados com LD.
Foram diagnosticados $16 / 33$ pacientes com NP versus 0 controles pareados por idade. Níveis de homocisteína (Hcy)também foram maiores em pacientes com $\mathrm{NP}(\mathrm{n}=16)$. O polimorfismo COMT A158G foi demonstrado, favorecendo baixa atividade enzimática em pacientes com NP versus controles sem NP.

Os estudos eletrofisiológicos mostraram que 93\% (26/28) dos pacientes com HIN apresentaram anormalidades eletrodiagnósticas, incluindo $75 \%(21 / 28)$ com neuropatia sensório-motora leve, $18 \% \quad(5 / 28)$ com características eletrofisiológicas da síndrome do túnel do carpo; enquanto apenas 7\% (2/28) dos achados normais. Conclusão: NP induzida por homocisteina é NP de fibras grossas com c desmielinização e desnervação axonal.

A prevalência de NP em pacientes com DP foi 35\%. Os níveis de MMA mostraram correlação positiva com TNSr e KNPQ em pacientes com DP com NP enquanto a VB12 e a homocisteína não apresentaram correlação estatisticamente significativa

Conclusão: MMA sérico correlacionou-se positivamente com a gravidade da dor neuropática e isso pode ser usado como marcador útil na avaliação da NP na DP.

A prevalência total de NP foi de $49,3 \%$ no grupo de estudo. Nos pacientes em uso de LD , a frequência de NP foi de 67,3\% versus no grupo sem uso de LD ( em uso de outras terapias dopaminérgicas) onde nenhum sujeito apresentou NP. No grupo L-Dopa-NP, 10/35 (28,57\%) apresentaram modificações compatíveis com NP axonal sensitiva, $10(28,57 \%)$ apresentaram NP axonal sensitiva moderada e 15 apresentaram perda axonal sensitiva grave e perda motora axonal Concluño: A NP é mais frequente en pacientes tratos com LD. Una Concrũa: A NP é mis frequene en pacientes tratos com LD. Uma exposição mais longa a doses elevadas de LD pode causar desequilíbrio do

A frequência de NP em pacientes com DP é maior que nos controles. No grupo $\mathrm{DP}$, indicadores clínicos e neurofisiológicos da NP, manifestados como neuropatia axonal simétrica e predominantemente sensorial, foram mais frequentes do que no grupo controle e observados em $43,1 \%$ vs. $13,7 \%$ e $15,7 \%$ vs. $2 \%$ dos indivíduos, respectivamente . A presença de NP correlacionou-se com a idade e a gravidade da DP. Estes também apresentaram nível mais alto de Hcy versus pacientes com DP sem NP, porém a diferença não foi estatisticamente significante. Os achados pela ENMG pode sugerir NP de pequenas fibras (SFN) como a forma dominante em pacientes com DP. 


\begin{tabular}{|c|c|c|}
\hline 11 & & \\
\hline $\begin{array}{l}\text { Mathukumalli et al., } \\
2020\end{array}$ & Estudo transversal & $\begin{array}{l}\text { Foram avaliados } 93 \text { pacientes com DP e } 70 \text { controles } \\
\text { sem DP. Foram realizados testes de soro B12, } \\
\text { homocisteína, folato, EMG e função autonômica. }\end{array}$ \\
\hline Nolano et al., 2017 & Estudo transversal & $\begin{array}{l}\text { Oitenta e cinco pacientes ( } 49 \text { homens, idade } 61,3+/-9,7 \\
\text { anos) com DP sem sinais eletrofisiológicos de NP. } 48 \\
\text { participantes estavam livre do uso de qualquer trata- } \\
\text { mento e } 37 \text { em uso de LD. Foram submetidos a exames } \\
\text { clinico-neurológico e ENMG. } \\
\text { A inervação sensitiva e autonômica foi visualizada com } \\
\text { anticorpos específicos e analisada por microscopia } \\
\text { confocal. Os dados foram comparados com os obtidos } \\
\text { em controles saudáveis comparáveis ao sexo e à idade. } \\
\text { Em } 35 \text { pacientes, biópsias de pele foram realizadas } \\
\text { bilateralmente para avaliar as diferenças lado a lado. }\end{array}$ \\
\hline Ceravolo et al., 2013 & Estudo multicêntrico & $\begin{array}{l}\text { Um estudo multicêntrico de } 330 \text { pacientes com DP e } \\
137 \text { controles saudáveis com distribuição etária compa- } \\
\text { rável foi realizada. Com respeito exposição à levodopa, } \\
144 \text { pacientes tiveram longa exposição } \\
\text { ( } 3 \text { anos) a levodopa (LELD), } 103 \text { pacientes tiveram } \\
\text { exposição curta ( }<3 \text { anos) à levodopa (SELD) e } 83 \\
\text { pacientes não teve exposição à levodopa (NOLD). A } \\
\text { função nervosa era avaliado usando o escore reduzido } \\
\text { de neuropatia total. estudos neurológicos sensoriais } \\
\text { antidrômicos e de condução do nervo fibular foram } \\
\text { realizados por neurofisiologistas cegos para a existência } \\
\text { de neuropatia clínica características ou tratamento de } \\
\text { DP }\end{array}$ \\
\hline 14 & & \\
\hline Merola et al., 2016 & Estudo transversal & $\begin{array}{l}\text { Foram avaliados prospectivamente } 33 \text { pacientes, de } \\
\text { ambos os sexos, portadores de DP em uso de infusão } \\
\text { intestinal de gel de LD. Foram submetidos a avaliação } \\
\text { clínica/neurologica, bioquímica e eletrofisiológica. }\end{array}$ \\
\hline
\end{tabular}

Avaliar a prevalência de
deficiência de vitamina B12, hiper-homocisteinemia pem sua associação com a NP

Estudar a patologia das fibras pequenas e grandes em pacientes não tratados com drogas e tratados com L-dopa afetada pela doença de Parkinson (DP) nas fases iniciais, antecedendo ocorrência de anormalidades eletrofisiológicas neuropáticas.

Avaliar o risco de neuropatia em pacientes com doença de Parkinson (DP) e avaliar o papel da levodopa exposição como um fator de risco potencial.

Relatar a incidência de da NP em pacientes tratado com gel de LD em infusão intestinal.
Níveis sérios de B12 e homocisteína não foram diferentes entre os casos e controles. Sete dos $93(9,68 \%)$ pacientes com DP tinham NP. Os valores medianos dos níveis séricos de B12, folato e homocisteína em pacientes com ou sem NP não puderam ser comparados, pois apenas sete de nossos pacientes inham NP. Conclusão: A prevalência de deficiência de vitamina B12, hipero: A preva Ancia de deficiência de vitá mina B12, hiperhom comparada à população ocidenta Se a NP em pacientes con DP pode secundária à deficiência de B12 / hiper-homocisteinemia é uma especulação.

Foi detectada uma redução de fibras nervosas intra-epidérmicas em pacientes com DP versus controles . Esta foi maior no lado mais afetado $(\mathrm{p}<0,01)$ Apresentaram ainda limiares tácteis e térmicos aumentados, comprometimento da percepção mecânica da dor e produção reduzida de suor . Os grupos virgens de tratamento com LD diferiram apenas na densidade corpórea de Meissner Conclusões: A patologia das fibras finas e grossas ocorre nos estágios iniciais da DP e pode ser responsável pelo comprometimento sensitivo e autonômico.

No geral, $19,40 \%$ dos pacientes em grupo LELD, $6,80 \%$ no grupo SELD, 4,82\% no grupo Grupo NOLD e $8,76 \%$ no grupo controle foram diagnosticados com neuropatia (axonal, predominantemente sensorial). O risco de neuropatia foi 2,38 maior no grupo LELD do que no grupo

rupo controle. Em uma comparação entre pacientes com e sem neuropatia (teste $\mathrm{t}$ de Student), a dose de levodopa foi maior ( $\mathrm{P}<0,0001)$, os níveis sérico de vitamina B12 foram menores (P 50,0102 ), e os níveis de homocisteína foram maiores $(\mathrm{P}<0,001)$ nos pacientes com neuropatia. Nossos resultados demonstrar que a duração da exposição à levodopa, juntamente com a idade, é o principal fator de risco para o desenvolvimento de neuropatia.

Na linha de base (antes do início da terapia com LCIG), 3/33 (9\%) pacientes apresentaram NP sintomática e 7/33 (21\%) subclínico . Durante o seguimento de 24,36 +/- 12,18 meses, 2/23 (8,7\%)pacientes com avaliação clínicoeletrofisiológica basal normal desenvolveram um NP subaguda, $2 / 23(8,7 \%)$ desenvolveram um NP crônico e 7/23 (30,4\%),NP subclínica. 12/23 pacientes pacientes não desenvolveram alterações clínicas e / ou eletrofisiológicas (sem NP).Os níveis de homocisteina também se mostraram elevados em relação aos valores basais. Enquanto nenhuma alterado evolutiva foi observada nos níveis de vitamina B12 ou folato.Não foi encontrada correlação entre UPDRS modificações no escore e alterações nas escalas clínicas específicas para NP Conclusão: NP representa característica subestimada da DP avançada, provavelmente relacionada a origens multifatoriais da exposição a longo prazo à 


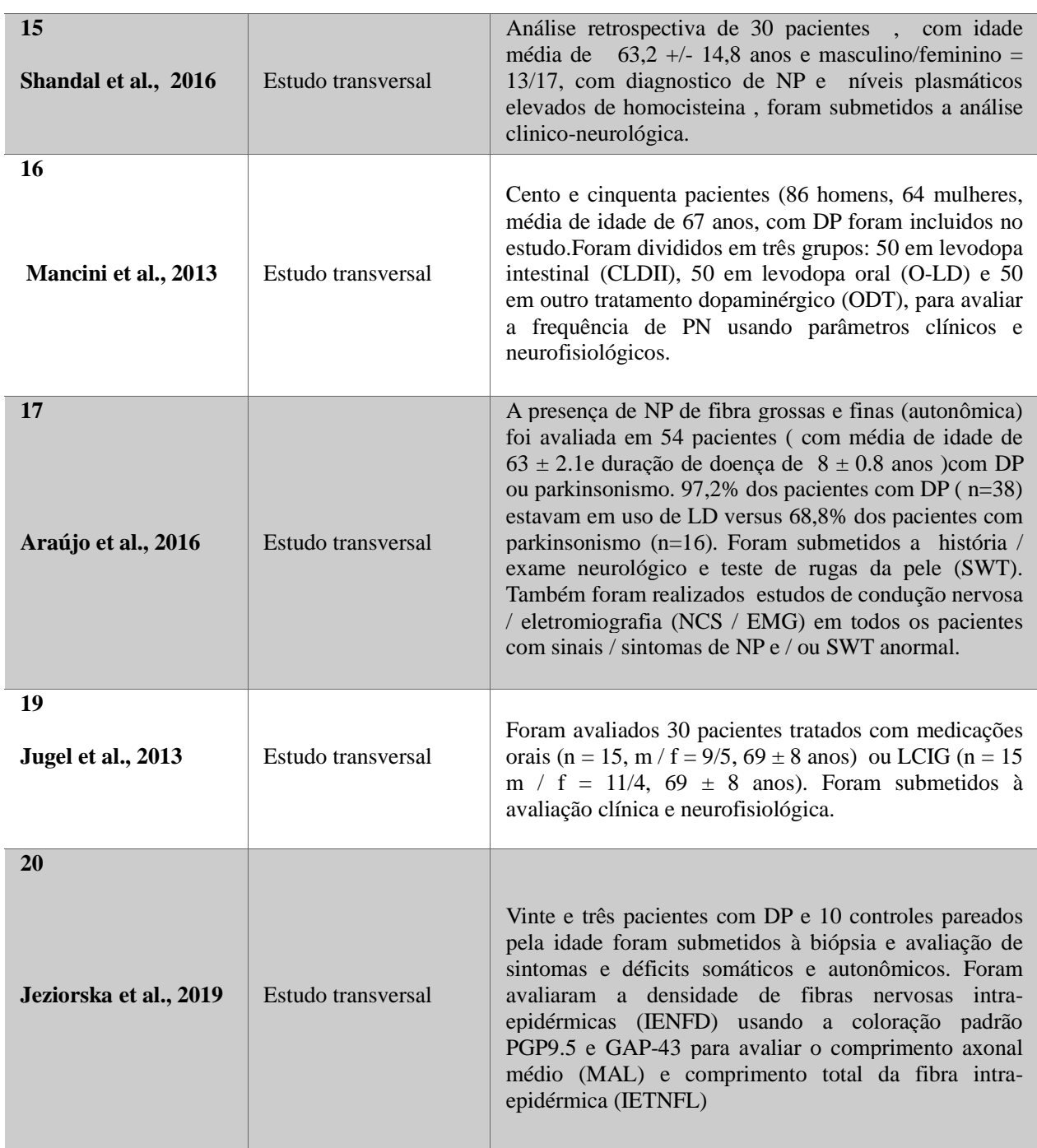

Caracterizar as características clínicas da NP induzido pelos níveis sérios elevados de homocisteína.

Comparar a frequência e características da NP em pacientes com DP submetidos a diferentes regimes terapêuticos.

Avaliar a presença de NP de fibra grossas e finas (autonômica) em 54 pacientes com DP ou parkinsonismo em um ambulatório terciário do Brasil.

Investigar se há diferença na unção do nervo periférico entre pacientes com tratamento oral versus infusão intestinal de gel de Levodopa / Carbidopa.

Estabelecer a extensão da degeneração e regeneração de IENF e sua relação com déficits clínicos/neurológicos na DP.
Todos tiveram aumento isolado dos níveis de homocisteína e níveis normais de B12, folato e ácido metilmalônico. Déficits sensitivos distais estavam presentes em 18/30 (60\%) pacientes, sendo este o sintoma predominante.

A frequência de NP sem causa evidente foi de $28 \%$ no CLDII, 20\% no O-LD e $6 \%$ nos pacientes com ODT. Clinicamente, $71 \%$ dos pacientes com CLDII e odos os pacientes com NP O-LD e ODT apresentaram PN sensorial subaguda.

Por outro lado, $29 \%$ dos pacientes com DCLII apresentaram NP motora aguda. Dose diária de LD, VB12 e níveis de homocisteína (hcy) diferiram significativamente nos pacientes com NP versus pacientes sem NP.

Conclusões: O presente estudo apoia relação entre LD e NP e confirma haver desequilíbrio nos nineis serviços de VB12 e hcy.

SWT foi realizado em 48 pacientes (33 PD, 15 parkinsonismo). No grupo com DP, o SWT foi anormal em 57,6\% dos pacientes testados (compreendendo 50\% de todos os pacientes com DP). No grupo parkinsonismo, o SWT foi anormal em 37,5\% (compreendendo 35,3\% do grupo parkinsonismo). NCS / EMG foi realizado em 39 pacientes (26 DP e 13 parkinsonismo). Doze das 26 DP (34,2\% dos todos os DP) e 4 dos 13 parkinsonismo (23,5\% do grupo parkinsonismo) apresentaram resultados anormais de NCS / EMG. Prevalência de NP foi semelhante nos grupos de DP e parkinsonismo detectados por NCS / EMG ou SWT. Conclusões: Fibras grandes e fibras pequenas (autonômicas) são comuns em pacientes com DP e parkinsonismo.

NP axonal foi comum em ambos os grupos. porém mais graves nos pacientes ratados com infusão intestinal de gel de Levodopa / Carbidopa em relação aos pacientes tratados com LD oral

conclusão: NP axonal em pacientes em infusão intestinal de LD pode estar associada às doses altas de LD associadas à terapia e / ou aos efeitos desnutricionais da aplicação intestinal.

IENFD, MAL e IETNFL foram significativamente reduzidos em pacientes com DP versus controles. IENFD correlacionou-se significativamente com a duração da doença, dose cumulativa de levodopa, Escala Unificada de Avaliação de Doenças de Parkinson, Parte III (UPDRS-III), Schwab e England Activities of Daily Living (ADL) NSP e proporção 30:15 O IETNFL / Á correlaciving (ADL) , Ne cOm a Parkinson (SCOPA-AUT) e (DNS) e o (DNS) e O IETNFL / Comprimento correlacionado com o DNS. A MAL correlacionou-se com SCOPA-AUT, DNS e DB-HRV. Conclusão:Aumento da degeneração de IENF e diminuição da regeneração se correlacionam com sintomas somáticos e autonômicos e déficits em pacientes com DP. 
21

Mu et al., 2013

Estudo transversal

(22)

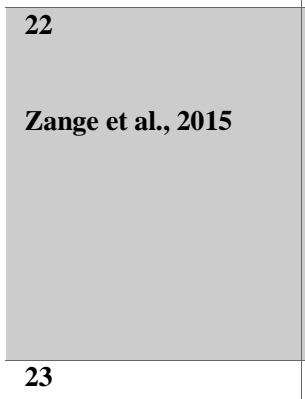

Estudo transversal

Foram realizadas biopsia de pele de 10 pacientes com atrofia de múltiplos sistemas (AMS) , $10 \mathrm{com}$ DP, juntamente com seis indivíduos controle com

Os nervos sensoriais que supriam a faringe foram diagnóstico clínico e DP neuropatologicamente confirmada $(\mathrm{n}=10)$ e faixa etária saudável controles (n = 4). Examinamos o nervo glossofaríngeo (nervo craniano IX), o ramo sensitivo da faringe do nervo vago (PSB-X) e o nervo laríngeo superior interno (ISLN) que ins a laringofaringe. A imuno-histequenica sinucleína fosforilada foi usada para detectar patologia de Lewy.

tremor essencial. Foram examinados por imunohistoquímica depósitos de SNCA fosforilados nas fibras nervosas simpáticas da pele e a densidade das fibras nervosas dérmicas foi avaliadas.

Vinte e oito pacientes com DP (16 homens, com média de idade e desvio padrão de 65,6+/-10,7 anos) livre de sintomas neuropáticos e distúrbios sistêmicos versus 23 controles de mesma idade e sexo ( 12 homens, com média de idade e desvio padrão de 65,1 +/-9,9 anos). Foram realizadas biópsia de pele, potencial evocado pelo calor por contato (CHEP) e testes sensitivos quantitativos (QST) para

A durac̃a A duraça da DP foi de 7,1 3,2 (variac̃o de 2 a 17 anos) 25,6+/- 9,7 (faixa de 10 a 48) durante o período off.

\begin{tabular}{|l|l|l|}
\hline 24 & $\begin{array}{l}\text { Foram estudados 26 pacientes com DP e 22 controles } \\
\text { usando o exame neurológico e a avaliação } \\
\text { estudos de condução (ECN). Também foram realizadas } \\
\text { a Escala de Neuropatia Precoce de Utah (UENS) e } \\
\text { biópsia de pele e microscopia confocal da córnea } \\
\text { (CCM). }\end{array}$ \\
\hline
\end{tabular}

Investigar o envolvimento dos via termonociceptiva na DP.

Determinar se a neuropatia periférica ocorre na DP inicial não tratada.
Agregados axonais de sinucleína nos nervos sensoriais da faringe foram identificados em todos os indivíduos com DP, mas não em controles. A densidade de lesões positivas a-sinucleína foi maior em pacientes com DP con disfagia versus aqueles sem disfagia. Além do que, além do mais, $>$ inucleína As fibras nervosa imunorreativas no ISLN eram muito mais abundantes que os do nervo craniano IX e PSB-X. Estes achados sugerem que os nervos sensoriais da faringe são diretamente afetados por processos patológicos na DP. Essas anormalidades podem diminuir a faringe sensação, comprometendo assim os reflexos de deglutição e de proteção das vias aéreas e contribuindo para disfagia e aspiração

eitos relacionados à DP a nervos sensoriais periféricos inervando a faringe.

Testar a hipotese de que a alfaNCA pode ser detectada em nervosas simpáticas Parkinson, porém não na Atrofia de Múltiplos Sistemas.

Todos os pacientes com DP expressaram SNCA fosforilado nas fibras nervosas simpáticas cutâneas, correlacionando-se com denervação independente da idade dos elementos autonômicos da pele. Em contraste, nenhum SNCA fosforilado foi encontrado nas fibras nervosas autonômicas da pele de pacientes com atrofia de múltiplos sistemas e indivíduos com controle de tremor essencial. Esses achados apóiam que a deposição de SNCA fosforilada é causadora da degeneração das fibras nervosas na doença de Parkinson. Além disso, a

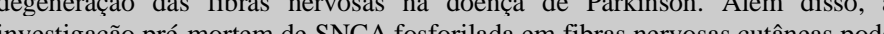
( diferenciar a doença diferenciar a doença de Parkinson de atrofia de múltiplos sistemas.

Os pacientes com DP apresentaram redução intra-epidérmica da densidade de fibras nervosas e amplitude de CHEP. Vinte e três pacientes $(82,1 \%)$ apresentaram densidades anormais de IENF e $18(64,3 \%)$ apresentaram CHEP anormal. Nove $(32,1 \%)$, apresentaram limiares térmicos anormais nos pés. No total, 27 pacientes $(96,4 \%)$ apresentaram pelo menos 1 anormalidade no IENF, CHEP ou limiares térmicos do pé, indicando disfunções de fibras finas. Nos controles , a amplitude de CHEP correlacionou-se linearmente com a densidade de IENF . Essa relação desapareceu na DPe a amplitude de CHEP foi negativa . Esa recocio do negativamente correlacionada com gravidade da DP independente da idade, centrais na sistemas termonociceptivos. Conclusão: $O$ presente estudo sugere que o comprometimento de fibras finas sensitivas nos níveis periférico e central é uma característica intrínseca da DP.

Não foram encontradas diferenças significativas entre os grupos, exceto por algumas alterações de sensibilidade vibratória no grupo DP. As densidades de fibras nervosas epidérmicas nas biópsias da pele foram semelhantes entre as

coortes. No entanto, usando CCM , teste mais sensível e marcador substituto de danos às fibras fibras na NP, descobrimos que pacientes com DP apresentam menor densidades e comprimentos de fibras nervosas da córnea comparado aos controles. Conclusões: Nossos resultados positivos no CCM fornecem evidências de PN pré-clínica em pacientes recém diagnosticados com DP. 
25

Edwards et al., 2012

Estudo transversal

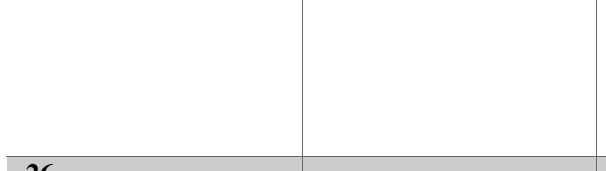

\begin{tabular}{|l|l|}
\hline 26 \\
Zhang et al., 2019 & Estudo transversal \\
\hline 27 & \\
\hline Doppler et al., 2014 & Estudo transversal \\
\hline & \\
\hline
\end{tabular}

Zhang et al., 2020
Duzentos e trinta e um indivíduos com diabetes mellitus (DM) com NP leve ou inexistente e 6 controles foram submetidos à avaliação da neuropatia diabética (DPN)por um escore de sintomas e incapacidade neuropatia Testes com monofilamento de $10 \mathrm{~g}$ testes seur. patia, Testes con comprimento das fibras nervosas da córnea, a densidade comprimento das fibras nervosas da córnea, a densidade
dos ramos e tortuosidades foram medidos usando dos ramos e tortuosidades foram medidos usando
microscopia confocal. Diferenças na morfologia do microscopia confocal. Diferenças na morfologia do
nervo corneano entre indivíduos com e sem DPN e os nervo corneano entre indiví́
controles foram investigados

Foram realizadas avaliações clínicas e biópsia do nervo sural para avaliar as características clínicas e os deposição de $\alpha$-sinucleína total (tSNCA) e pSNCA em peças de biópsia usando métodos de imunoquímica em 16 PD pacientes e 15 controles. Além disso, a coloração por imunofluorescência foi realizada usando certos anticorpos para caracterizar o componente do nervo sural e localizar a expressão de pSNCA.

Trinta e um pacientes com DP e 35 controles foram submetidos à biopsia de pele e quantificados em relação a colocalização da alfa-sinucleína fosforilada

nas fibras nervosas somatossensitivas e autonômicas bem como o padrão de perda de diferentes subtipos de fibras dérmicas.

Quatorze pacientes com DP (incluindo 5 com distúrbios sensitivos e 9 sem distúrbios sensitivos) e 6 controles. Foi realizado estudo eletrofisiológico do nervo sural. Com amostras de biópsia do nervo sural, foram realizadas alterações ultraestruturais do nervo e realizadas alterações ultraestruturais do nervo e
observado por microscopia eletrônica; Proteína de ácido observado por microscopia eletrônica; Proteína de ácido fibrilar glial de biomarcador de células de Schwann
(GFAP) e citocinas inflamatórias, incluindo interleucina-1beta (IL-1 $\beta$ ), interleucina 6 (IL6) e fator de necrose tumoral alfa (TNF- $\alpha$ ) foram detectadas por imuno-histoquímica, e o resultado da imunocoloração foi avaliada semiquantitativamente.
Avaliar a morfologia do nervo corneano sub-basal como marcador oftálmico para a detecção de DPN.

ara a O comprimento da fibra nervosa da córnea foi significativamente reduzido em diabéticos sem DPN A densidade de ramo do nervo corneano fo controles. onclusar cínich DPN

Deposição de pSNCA foi encontrada em 16/16 pacientes com DP, com um percentual positivo alto de $100 \%$, mas em 0/15 controles, no entanto, todas as peças de biópsia mostraram resposta positiva à coloração imuno-histológica de tSNCA no nervo fibras. O pSNCA foi expresso principalmente nas células de Schwann, mas dificilmente nos axônios, demonstrando um novo padrão de Expressão de pSNCA no sistema nervoso periférico. Conclusão: Os achado sugerem que o nervo sensitivo periférico também está envolvido na patologia do SNCA n DP. A PSNCA no nervo sural pode sevir como um novo biomareado para o diagnóstico precoce de DP :

Depósitos de alfa-sinucleína fosforilada foram identificadas em 16/31 Pacientes com DP, mas em controles $0 / 35$. A quantificação das fibras nervosas revelou

Caracterizar a deposição de alfa-sinucleína em nervos cutâneos de pacientes com DP e verificar se o envolvimento do nervo periférico é uma característica intrínseca à DP . is tipos de neurodegeneração periférica na DP: (1) uma redução dependente do comprimento de fibras finas intraepidérmicas pequenas e (2) uma severa redução não dependente do comprimento das fibras nervosas intraepidérmica munorreativas da substância P . As alterações histológicas não se correlacionaram a deficiência de vitamina B12. Conclusão: O estudo sugere que a perda de fibras nervosas periféricas é uma característica intrínseca da DP e que as alterações do nervo periférico podem refletir os dois tipos patologia central da DP relacionada à alfa-sinucleína, morte neuronal e degeneração axonal. A deteç̧ão de alfa-sinucleína fosforilada nas fibras nervosas dérmicas pode ser um teste diagnóstico útil para DP com alta especificidade, mas baix sensibilidade

Caracterizar a ativação das Comparado com controles saudáveis, a velocidade de condução nervosa (NCV) células de Schwann (SCs) e o diminuiu a amplitude do potencial de ação dos nervos para baixo e sensorial umento da expressão de (SNAP) diminuiu em pacientes com DP, acompanhada de degeneração axonal e itocinas inflamatórias IL-1 $\beta$, lesões desmielinizantes e expressão de GFAP e citocinas inflamatórias -6 TNF- $\alpha$ no nervo sural da aumentaram. Conclusõo: existe lesão nas vias sensitivas periféricas en DP, e explorar ainda mais se a pacientes com DP, acompanhada por ativação e inflamação das células de inflamação do nervo periférico Schwann demonstram assim que a inflamação do nervo periférico participa do a causa dos distúrbios ensoriais da DP. processo fisiopatológico da DP e isso pode estar relacionado a distúrbios sensitivos. 
Research, Society and Development, v. 10, n. 7, e35410716813, 2021

(CC BY 4.0) | ISSN 2525-3409 | DOI: http://dx.doi.org/10.33448/rsd-v10i7.16813

29

Lee \& Baik, 2020

Estudo transversal
105 pacientes com DP, com critérios do Banco de

Cérebro do Reino Unido para diagnóstico clínico de DP,

e não medicados. O protocolo consistiu em avaliar as

características clínicas da doença, sexo, idade, tempo de

doença, aspectos fenotípicos e aplicar as escalas de

avaliação. Como estadiamento de Hohen \& Yahr e

URRS-III. Alch disso, forn realizados exames de

eletroc

condução motora e sensorial e exames laboratoriais,

para análise dos níveis dos metabólitos séricos de

vitamina B12, homocisteína e acido úrico.
Dos 105 pacientes, 24 apresentavam e velocidade de condução nervosa alterada.

Investigar a prevalência de europatia periférica pacientes com DP e sua relaça (én de metabólitos séricos

homocisteína, vitamina B12 uratos.

Destes 20 pacientes apresentaram um tipo de sensorial e motora combinado, enquanto três eram tipo de sensorial puro e um era motor puro. Nove pacientes inham síndrome do túnel do carpo. O grupo PD com NP demonstrou níveis séricos mais elevados de homocisteína e ácido úrico em comparação com o

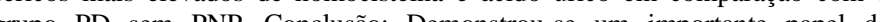
vitamo 12 . vitamina B12, homocisterna e acido únco na patogenese da NP en pacientes com DP, sugerindo-se envolvimento metabólico na deterioração do sistem nervoso periférico na DP. 


\section{Discussão}

\section{Neuropatia periférica e uso crônico de levodopa}

Dois estudos prospectivos e transversais analisaram amostras homogêneas de pacientes, com DP em uso de LD versus controles sem DP e sua possível relação com os niveis plasmáticos de ácido metilmalônico, através de dados clínicos e eletrofisiológicos, além de correlacionar a exposição à levodopa, a duração da DP, a gravidade da neuropatia e o papel da vitamina B12. No primeiro foram comparados 37 pacientes controles sem DP versus 37 com DP de mesma idade e sexo em relação à prevalência de NP. No segundo, 58 pacientes com DPI versus 58 controles. Ambos os estudos mostraram uma prevalência relativamente alta de NP, variando de 37,8 a 55\%, em comparação com 8,1 a $9 \%$ de frequência nos controles ( Rajabally \& Martey, 2011; Toth et al. 2010), ou seja, aqueles indivíduos com DP apresentavam maior prevalência de NP do que os controles. Demonstrou-se que a neuropatia é mais prevalente em indivíduos com DP. Observou-se que aqueles indivíduos em uso de levodopa, com DP e NP, apresentavam altos níveis plasmáticos de AMM e Hcy, sendo que a gravidade da NP apresentava proporção direta com o tempo de exposição e os níveis sangüineos de levodopa, assim como de AMM. Embora a causalidade não esteja bem estabelecida, a exposição à levodopa está associada com a neuropatia sensitivo-motora e a elevação da AMM em pacientes com DP. Apesar dos autores sugerirem que ocorram mudanças no metabolismo da vitamina B12 (VB12) quando da manifestação de NP, não existe consenso de que seja um bom biomarcador para fins de diagnóstico. Sabe-se que a conversão de Levodopa (LD) em dopamina requer doação de grupo metil fornecido pela adenosilmetionina, sendo que tal reação leva à formação de hcy. A remetilação subsequente de hcy requer VB12 como cofator, e formas alternativas de degradação requerem metil tetra-hidrofolato e piridoxina (vitamina B6). Desta forma, o uso crônico de LD ocasiona depleção de grupos metil e superprodução de hcy. Em vista disso, o consumo a longo prazo de LD, causa o acúmulo de hcy e, consequentemente, o esgotamento de vitaminas B6 e B12 (Andréasson et al., 2017; Paul et al., 2020). Outro fator agravante está relacionado à presença de hiper-homocisteinemia, que pode exercer efeitos, através de diferentes mecanismos, aumentando a vulnerabilidade a toxinas mitocondriais e radicais livres, induzindo reações inflamatórias e comprometimento dos mecanismos de reparo do DNA (Luo et al., 2013).

Tem-se observado que nos últimos anos houve aumento na prevalência de NP em pacientes com DP submetidos a exposição prolongada à levodopa (LD), apoiando-a como principal fator de risco (Vanta et al., 2019; Szadejko et al., 2016). Estudo de caso, com dois pacientes que desenvolveram neuropatia periférica axonal subaguda em tratamento com duodopa mostrou através da condução nervosa resultados compatíveis com polineuropatia sensitivo-motora axonal. $\mathrm{O}$ exame eletromiográfico da agulha da parte inferior extremidades (músculos tibiais anteriores) mostrou discretos sinais de desnervação com fibrilação e ondas agudas positivas. Já a análise do potencial de ação da unidade motora (MUAP) revelou aumento no número de potenciais polifásicos (> 20\%) e recrutamento máximo mostrou um número reduzido de potenciais (Mathukumalli et al., 2020). Por outro lado, a deficiência de VB12, por si só, não pode explicar todos os casos relatados de NP (Nolano et al., 2017). Em alguns indivíduos que apresentavam NP, os níveis plasmáticos de VB12 eram normais. Estudo transversal multicêntrico (330 DP x 137 controles), revelou um caso de NP subaguda e agravamento da NP preexistente em ausência de qualquer alteração nos níveis plasmáticos de VB12, Hcy ou MMA (Ceravolo et al., 2013). Esses estudos reforçam que a idade associada ao tempo de exposição à levodopa, são os principais fatores de risco para o desenvolvimento da NP.

Estudo prospectivo com 33 pacientes com DP, idades entre 55 e 78 anos, duração média da doença de 14,59 anos e uso de terapia intestinal com LD (dose de 1317,73 +/-456,25), realizou avaliação sob o ponto de vista clínico e eletrofisiológico, correlacionando incidência de NP versus alterações clinico-eletrofisiológicas, doses da terapia 
dopaminérgica, índice de massa corporal (IMC) e níveis plasmáticos de vitaminas do complexo B ( B1, B12 e folato) e homocisteína. Daqueles que iniciaram a terapia intestinal com LD, 3/33 (9\%) apresentavam NP sintomática e 7/33 (21\%) NP subclínica. Apenas 23/33 (70\%) apresentavam achados clínicos e eletrofisiológicos normais, sendo selecionados e incluídos nesta análise. Os critérios foram utilizados neste estudo para classificação de NP foram: NP simétrica definida como alteração de amplitudes e / ou velocidades do potencial de ação no estudo da condução nervosa em pelo menos dois nervos sensitivos, acompanhado de sinais e sintomas envolvendo o sistema nervoso periférico; piora durante o acompanhamento clínico definida por amplitude significativamente reduzida do potencial de ação ( $\geq 50 \%$ ) e / ou redução da velocidades de condução ( $\geq 20 \%$ ) em pelo menos dois nervos motores e/ou nervos sensitivos e 'NP subaguda' e 'NP Crônica' classificadas de acordo com a velocidade da progressão clínica. Aqueles que mostraram apenas alterações eletrofisiológicas foram classificados como NP subclínica. Durante o seguimento, dos pacientes selecionados, 2/23 (8,7\%) desenvolveram NP subaguda, $2 / 23$ (8,7\%) NP crônica, 7/23(30,4\%) NP subclínica e 12/23 $(52,2 \%)$ não desenvolveram NP. Diante disto, a terapia de infusão intestinal de gel de LD foi imediatamente descontinuada nos casos subagudos, porém não nos casos crônicos e formas subclínicas. Todos pacientes com NP foram suplementados com vitamina B1 e B12, evidenciando melhora clínica e / ou estabilidade substancial. Em comparação com a NP subaguda, a NP crônica se desenvolveu após um período de acompanhamento mais longo, sendo correlacionada com os níveis plasmáticos de homocisteína e doses diárias equivalentes mais altas de LD. Nenhum fator causal que justifique claramente as formas subagudas foi reconhecido, enquanto a neurotoxicidade mediada pela homocisteína parece estar subjacente à patogênese das formas crônicas (Merola et al., 2016). Provavelmente, esses casos podem estar relacionados à neurotoxicidade da homocisteína induzida pela levodopa, resultando no acúmulo de radicais livres, aumento da vulnerabilidade a toxinas mitocondriais e comprometimento do mecanismo de reparo do DNA (Shandal \& Luo, 2016). Diante do exposto, é aconselhável manter acompanhamento clínico-neurofisiológico de todos pacientes com DP em tratamento com levodopa e avaliar periodicamente os níveis séricos de homocisteína e vitamina B12.

\section{Neuropatia periférica e progressão da Doença de Parkinson}

Apesar dos relatos sobre o aumento na prevalência de NP em pacientes com DP, a maioria estava em uso de LD ( Mancini et al., 2014; Araújo et al.,2016; Jugel et al., 2013). Diante disso, embora tenha-se sugerido como hipótese que o uso prolongado de L-dopa, direta ou indiretamente, cause neuropatia, não se pode descartar que outros fatores, tais como mutações genéticas, envelhecimento ou a própria DP "isoladamente", possam causar danos aos sistema nervoso periférico (SNP) (Jeziorska et al., 2019; Zis et al., 2017). Muitos estudos têm investigado os danos intrínsecos ao SNP causados pelo tempo de doença e gravidade da DP demonstrando comprometimento do mesmo.

O envolvimento do nervo periférico pela DP pode ocorrer ao longo dos anos de comprometimento da doença. Em seu estudo, detectaram agregados de $\alpha$-sinucleína nos ramos motores e sensitivos do nervo vago, nervo glossofaríngeo e nervo laríngeo superior interno em pacientes com DP e disfagia (Mu et al (2013) Outros estudos detectaram depósitos de alfa-sinucleína fosforilada e neuropatia de fibras finas em pacientes com PD ( (Zange et al., 2015; Lin et al., 2016). Estes achados não estavam presentes em controles saudáveis e em pacientes com outras síndromes parkinsonianas, como parkinsonismo vascular, tauopatias e portadores de mutação Parkin. Além disso, não diferença significativa em relação à duração da doença, exposição ao LD e fatores de risco para neuropatia, incluindo deficiência de VB12, ao se compararem os resultados encontrados na DP idiopática com outras síndromes parkinsonianas. Podgorny et al. (2016) estudaram uma coorte de 26 pacientes com DP e 22 controles sem DP, sendo os pacientes com DP recentemente diagnosticados, virgens de LD ou com exposição mínima (em uso de LD a menos de 3 
semanas), com idades entre 50 e 60 anos, em vários estágios da doença (Escala Hoehn e Yahr 1-2,5), para determinar se a NP é uma característica da DP antes de se instituir o tratamento. Além de testes padrão para NP, estudos de condução nervosa e exame físico, foram realizados testes mais sensíveis, incluindo biópsia de pele e microscopia confocal da córnea (MCC) para avaliar as fibras finas. Foram estudados usando exame neurológico e estudo eletroneurofisiológico. Nenhuma diferença significativa foi encontrada entre os grupos, exceto por um mudança na sensibilidade vibratória no grupo DP. A densidade de fibras nervosas epidérmicas nas biópsias de pele foi semelhante entre os grupos. No entanto, usando a microscopia confocal da córnea, um teste mais sensível e um marcador substituto de lesão de fibra fina, verificou-se que pacientes com DP tiveram uma redução significativa na densidade e comprimento das fibras em comparação aos controles. Neste estudo, evidências de NP pré-clínica em pacientes com DP precoce sugerem que a levodopa pode não ser o fator desencadeante da NP em pacientes com DP (Edwardset al., 2012; Podgorny et al., 2016). Já, em outro estudo, as alterações na velocidade de condução nervosa foram observadas em cerca de 22,8\% dos participantes apresentando, em sua maioria, comprometimento sensorial e motor associados. Ao mesmo tempo, ao se relacionarem os metabólitos plasmáticos, demonstrou-se níveis séricos mais elevados de hemocisteina e ácido úrico nos pacientes com DP e NP ( Lee \& Baik , 2020).

Zhang et al. (2019) realizaram avaliações clínicas e de biópsia do nervo sural, a fim de determinar as características de deposição de $\alpha$-sinucleína total (tSNCA) e $\alpha$-sinucleína fosforilada por pSNCA em amostras de biópsia usando métodos imunoquímicos em 16 pacientes com DP e 15 controles. No grupo DP, a idade média foi de 62 anos (+/- 10,5 anos). A coloração por imunofluorescência foi realizada usando certos anticorpos para caracterizar o componente do nervo sural e localizar pSNCA expressão. Em seus resultados, a deposição de pSNCA foi encontrada em 16/16 pacientes com DP com um porcentagem alta (100\% positivo) e nos controles 0/15. O pSNCA foi expresso principalmente em Schwann células, mas pouco nos axônios, demonstrando um novo padrão de expressão de pSNCA no sistema nervoso periférico.

O fato de depósitos de alfa-sinucleína terem sido observados no nervo sural de todos os pacientes com DP (com parestesia e também em alguns pacientes sem sintomas de parestesia) torna a biópsia desse nervo útil para o diagnóstico de DP, principalmente para os pacientes em estágio precoce da doença (Doppler et al., 2014). Além disso, a estrutura do nervo sural, unicamente composto por fibras sensitivas somáticas, sem fibras autonômicas, justifica os sintomas somatossensitivos em pacientes com DP e NP. O envolvimento expresso principalmente nas células de Schwann, por ativação e inflamação, através da lesão do nervo periférico, e o pouco comprometimento dos axônios sugere que o mecanismo fisiopatológico no SNP pode ser diferente do mecanismo do SNC (Zhang et al., 2020).

Em relação a etiologia da NP em pacientes com DP, deve ser considerado um aspecto mais amplo com características multifatoriais, podendo ser secundária a efeitos colaterais do tratamento, decorrer de doenças concomitantes associadas a NP, ou mesmo, de causas genéticas, que devem ser consideradas (Finsterer et al., 2021).

\section{Conclusões}

Apesar de estudos mostrarem maior prevalência de NP em indivíduos com DP em relação a controles de mesma idade e sexo, evidências mostram que a LD pode influenciar no desenvolvimento de NP em indivíduos com DP e seu uso prolongado parece atuar como fator causal. Embora estudos mostrem que a deficiência de vitamina B12 e os altos níveis séricos de homocisteina e acido malimalônico possam contribuir para o desenvolvimento da NP na DP, outros encontraram a presença de NP com níveis plasmáticos de Vit.B12 normais. A presença de alfa-sinucleina cutânea e nos nervos periféricos demonstra que esta organela não se deposita somente no sistema nervoso central, 
podendo ser considerada como doença multisistêmica. Diante disto, parece haver uma perspectiva multifatorial para desencadeamento da NP na DP e sua progressão, possivelmente por interação entre os fatores de risco ou efeitos comulativos. Sugere-se a realização de estudos prospectivos correlacionando o comprometimento do sistema nervoso periférico em pacientes com DP e o envolvimento dos distintos fatores de risco com a progressão e agravamento da sintomatologia desta doença.

\section{Referências}

Andréasson, M., Brodin, L., Laffita-Mesa, J. M., \& Svenningsson, P. (2017). Correlations Between Methionine Cycle Metabolism, COMT Genotype, and Polyneuropathy in L-Dopa Treated Parkinson's Disease: A Preliminary Cross-Sectional Study. J Parkinsons Dis., 7(4), 619-628.

Araújo, D. F., Melo Neto, A. P., Oliveira, I. S. C., Brito, B. S., Araújo, I. T., Barros, I. S., Lima, J. W. O., Horta, W. G., \& Gondim, F. A. A. (2016). Small (autonomic) and large fiber neuropathy in Parkinson disease and parkinsonism. BMC Neurol., 16, 139.

Barbosa, M. T., Caramelli, P., Maia C. D. P., Cunningham, M. C. Q., Guerra, H. L., Lima-Cost,a M. F., \& Cardoso, F. (2006). Parkinsonism and Parkinson's disease in the elderly: a community-based survey in Brazil (the Bambui study), Mov Disord., 21, 800-8.

Ceravolo, R., Cossu, G., di Poggio, M.,B., Santoro, L., Barone, P., Zibetti, M., Frosini, D., Nicoletti, N., Manganelli, F., Iodice, R., Picillo, M., Merola, A., Lopiano, L., Paribello, A., Manca, D., Melis, M., Marchese, R., Borelli, P., Mereu, A., Contu, P., Abbruzzese, G., \& Bonuccelli, U. (2013). Neuropathy and Levodopa in Parkinson's disease: evidence from a multicenter study. Mov Disord., 28:1391-7.

Cossu, G., Ceravolo, R., Zibetti, M., Arca, A., Ricchi, V. Paribello, P., Murgia, D., Merola, A., Romagnolo,, A., Nicoletti, V., Palermo, G., Mereu, A. , Lopiano, L. , Melis, M. , Abbruzzese, \& G., Bonuccelli, U. (2016). Levodopa and neuropathy risk in patients with Parkinson disease: Effect of COMT inhibition. Parkinsonism Relat Disord., 27:81-4.

Dickson, D. W. (2018). Neuropathology of Parkinson disease. Parkinsonism Relat. Disord., 46, S30-S33.

Doppler, K., Ebert, S., Uçeyler, N., Trenkwalder, C., Ebentheuer, J., Volkmann, J., \& Sommer, C. (2014) Cutaneous neuropathy in Parkinson's disease: a window into brain pathology. Acta Neuropathol., 128(1), 99-109.

Edwards, K., Pritchard, N., Vagenas, D., Russell, A., Malik, R. A., \& Efron, N., (2012). Efron N. Utility of corneal confocal microscopy for assessing mild diabetic neuropathy: baseline findings of the LANDMark study. Clin Exp Optom., 95(3):348-354.

Finsterer, J., Scorza, F. A., Scorza, C. A., \& Fiorini, A. C. (2021) Parkinson-related neuropathy. Clinics,), 76 : e2675.

Jeziorska, M., Atkinson, A., Kass-Iliyya L., Javed, S., Christopher Kobylecki, C., Gosal, D., Marshall, A., Silverdale, M., \& Malik R. A. (2019). Increased Intraepidermal Nerve Fiber Degeneration and Impaired Regeneration Relate to Symptoms and Deficits in Parkinson's Disease., Front Neurol., 10:111.

Jugel, C., Ehlen, F., Taskin B., Frank, T., Thomas, M., \& Klostermann M. F. (2013). Neuropathy in Parkinson's disease patients with intestinal levodopa infusion versus oral drugs. PLoS One, 8(6), e66639.

Lee, J. J., \& Baik J. S. (2020) Peripheral Neuropathy in de novo Patients with Parkinson's Disease, Yonsei Med J. 61(12):1050-1053.

Lin, C. H., Chao, C. C., Wu S. W., Hsieh, P. C., Feng F. P., Lin, Y. H., Ya-Mei Chen, Y. M., Wu, R. M., \& Hsieh S. T. (2016) Pathophysiology of Small-Fiber Sensory System in Parkinson's Disease. Medicine (Baltimore), 95 (10), e3058.

Luo, J. J., Bumanlag F., \& Dun, N. J. (2013). Electrophysiologic Features of Peripheral Neuropathy in Adults with an Isolated Elevated Plasma Level of Homocysteine. J Neurol Transl Neurosci., 2(1), 1027.

Mancini F., Comi C., Oggioni G. D., Pacchetti C., Calandrella, D., Moja, M. C., Riboldazzi, G., Tunesi, S., Dal Fante, M.., Manfredi, L., Lacerenza,. M., Cantello, R., \& Antonini, A. (2014). Prevalence and features of peripheral neuropathy in Parkinson's disease patients under different therapeutic regimens. Parkinsonism Relat Disord., 20 (1), 27-31.

Mathukumalli N. L., Kandadai M. R., Shaik J. A., Kanikannan M. A., \& Borgohain R. (2020). Serum B12, Homocysteine Levels, and their Effect on Peripheral Neuropathy in Parkinson's Disease: Indian Cohort. Ann Indian Acad Neurol., 23(1), 48-53.

Mendes, K. D. S., Silveira, R. C. C. P., Galvão, C. M. (2008). Revisão integrativa: método de pesquisa para incorporação de evidências na saúde e na enfermagem. Texto Contexto Enferm, 17(4), 758-64.

Merola A., Romagnolo A., Zibetti M., Bernardini A., Cocito D., \& Lopiano L. (2016). Peripheral neuropathy associated with levodopa-carbidopa intestinal infusion: a long-term prospective assessment. Eur J Neurol., 23, 501-9.

Mu L., Sobotka S., Chen J., Su, H., Sanders, I., Nyirenda, T., Adler, C. H., Shill, H. A., Caviness, J. N., Samanta, J. E., Sue., B. S., \& Beach, T. G., (2013). Parkinson disease affects peripheral sensory nerves in the pharynx. J Neuropathol Exp Neurol., 2(7), 614-623.

Nolano M., Provitera V., Manganelli F., Iodice R., Stancanelli, A., Caporaso, G., Saltalamacchia, A., Califan, F., Lanzillo, B., Picillo, M., Barone, P., \& Santoro, L. (2017), Loss of cutaneous large and small fibers in naive and l-dopa-treated PD patients. Neurology, 89, 776-84

Obeso, J. A., Stamelou, M., Goetz, C. G., Poewe, W., Lang, A. E., Weintraub, D., \& Stoessl, A. J. (2017). Past, present, and future of Parkinson's disease: A special essay on the 200th Anniversary of the Shaking Palsy. Mov Disord, 32(9), 1264-1310. 
Park J. S., Park D., Ko P. W., Kang K., \& Lee H. W. (2017). Serum methylmalonic acid correlates with neuropathic pain in idiopathic Parkinson's disease. Neurol Sci., 38(10), 1799-1804.

Paul D. A., Qureshi A. R. M., \& Rana A. Q. (2020). Peripheral neuropathy in Parkinson's disease. Neurol Sci., 41(10), $2691-2701$.

Podgorny P. J., Suchowersky O., Romanchuk K. G., \& Feasby T. E. (2016). Evidence for small fiber neuropathy in early Parkinson's disease. Parkinsonism Relat Disord., 28, 94-9.

Poewe W., Seppi1 K., Tanner C.M., Halliday, G. M., Brundin, P., Volkmann, J., Schrag. A. E., \& Lang, A. E. (2017). Parkinson disease. Nat Rev Dis Primers, 3(17013), 1-19.

Radhakrishnan D. M., \& Goyal V. (2018). Parkinson's disease: A review. Neurol India, 66, S26-S35.

Rajabally Y. A., \& Martey J. (2011). Neuropathy in Parkinson’s Disease. Prevalence and determinants. Neurology, 77, 1947-50.

Shandal V., \& Luo J. J. (2016). Clinical Manifestations of Isolated Elevated Homocysteine-Induced Peripheral Neuropathy in Adults. J Clin Neuromuscul. Dis. 17(3),106-109.

Szadejko K., Dziewiatowski K., Szabat K., Robowski, P., Schinwelski, M., Sitek, E., \& Sławek, J. (2016). Polyneuropathy in levodopa-treated Parkinson's patients. J Neurol Sci., 371, 36-41.

Toth C., Brown M. S., Furtado S., Suchowersky, O., \& Zochodne, D. (2008). Neuropathy as potential complication of levodopa use in Parkinson's disease, Mov. Disord., 23, 1850-1859.

Toth C., Breithaupt K., Ge S., Duan Y., Terris, J. M., Thiessen, A., Wiebe, S., Zochodne, D. W., \& Suchowersky, O. (2010). Levodopa, methylmalonic acid, and neuropathy in idiopathic Parkinson disease. Ann Neurol., 68, 28-36.

Tysnes O. B., \& Storstein A. (2017). Epidemiology of Parkinson's disease. J Neural Transm., 124(8), 901-905.

Vanta O. M., Tohanean N., Pintea S., \& Perju-Dumbrava L. (2019). Large-Fiber Neuropathy in Parkinson's Disease: Clinical, Biological, and Electroneurographic Assessment of a Romanian Cohort. J Clin Med., 8(10), 1533.

Zange L., Noack C., Hahn K., Stenzel, W., \& Lipp, A. (2015). Phosphorylated $\alpha$-synuclein in skin nerve fibres differentiates Parkinson's disease from multiple system atrophy. Brain., 138(Pt 8), 2310-2321.

Zhang H, Zhu L, Sun L, Zhi Y., Ding, J., Yuan, Y. S., Shen, F. F., Li, X., Ji, P., Wang, Z., Niu, Q., \& Zhang, K.Z. (2019). Phosphorylated $\alpha$-synuclein deposits in sural nerve deriving from Schwann cells: A biomarker for Parkinson's disease. Parkinsonism Relat Disord. 60:57-63.

Zhang H, Wu J., Shen F. F., Yuan, Y. S., Li, X., Ji, P., Zhu, L. Sun, L., Ding, J., Niu, Q., \& Zhang, K.Z. (2020). Activated Schwann cells and increased inflammatory cytokines IL-1 $\beta$, IL-6, and TNF- $\alpha$ in patients' sural nerve are lack of tight relationship with specific sensory disturbances in Parkinson's disease. CNS Neurosci Ther, 26(5), 518-526.

Zis P., Grünewald R. A., Chaudhuri R. K., \& Hadjivassiliou M. (2017). Peripheral neuropathy in idiopathic Parkinson's disease: A systematic review. J Neurol Sci., 378, 204-209 